\title{
TUMOURS OF THE TEMPORAL LOBE OF THE BRAIN AND THEIR TREATMENT.
}

$B y$ CECIL P. G. WAKELEY,

D.Sc., F.R.C.S., F.R.S.E., F.A.C.S., F.R.A.C.S. (Hon.)

(Fellow of King's College, London. Senior Surgeon King's College Hospital and the West End Hospital for Nervous Diseases. Consulting Surgeon to the Maudsley Hospital and to the

Royal Navy. Hunterian Professor Royal College of Surgeons of England.)

It may be said quite definitely, that the temperosphenoidal lobe of the brain is too large an area for any definite signs and symptoms to follow lesions within it. The temporal lobes still remain more or less silent areas of the brain.

\section{Symptomatology.}

Tumours of the temporal lobes may, like intracranial tumours elsewhere, give rise to both general and local signs. From a diagnostic point of view, the temporal lobe falls into the category of one of the "silent" areas of the brain, in the same way as the frontal lobes. Large lesions can arise in this situation and give rise to no localising signs whatever. This, of course, makes for great difficulty in early diagnosis, and in early successful treatment.

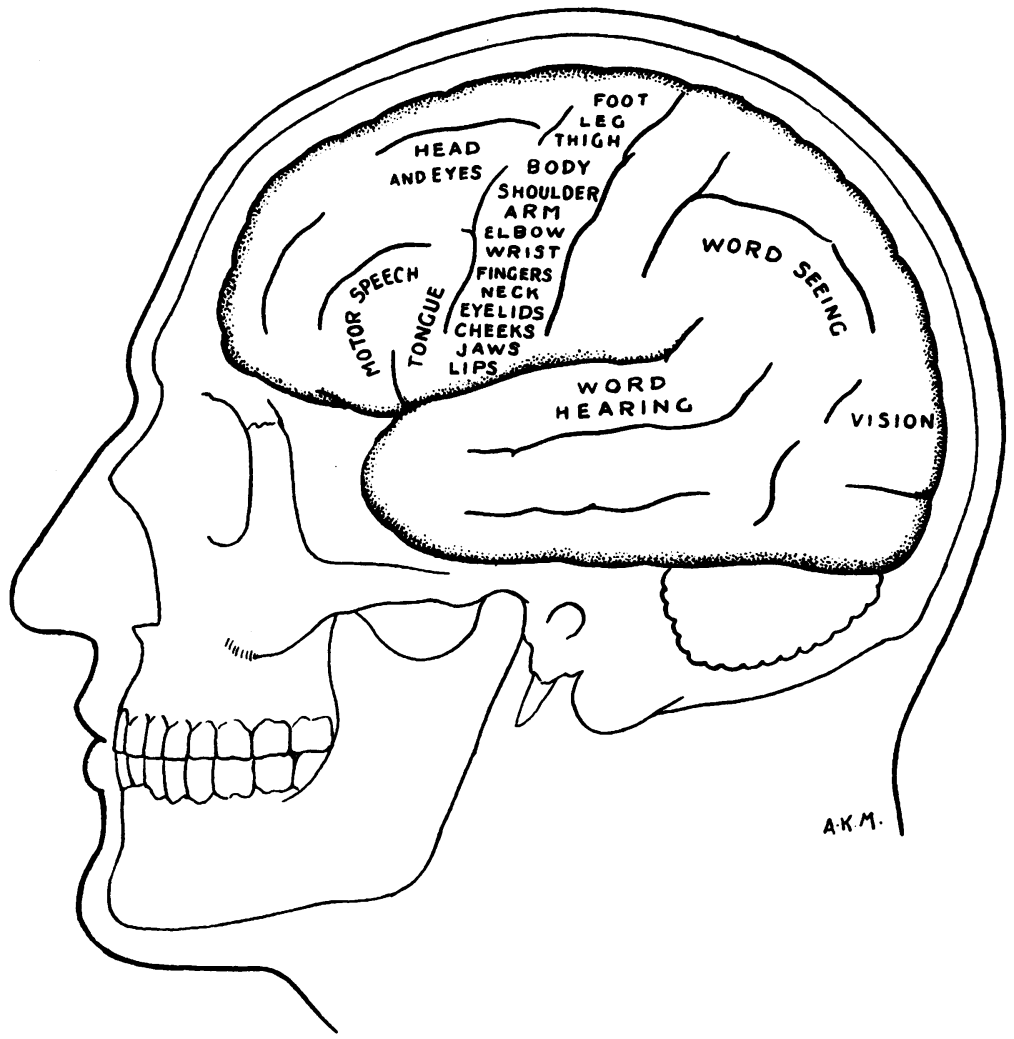

FIG. I.-Drawing showing the anatomical position of the temporal lobe and the associated areas of the cortex.

The local signs produced are those which are due to the actual destruction of nerve cells and fibres, either by the growth itself, or by the vascular and œedematous changes which surround the growth. The general signs are those due to the rise in 
intracranial pressure from distortion of the ventricular system, vascular congestion, and actual displacement from the added volume of the growth. The local signso can only be studied by reference to the anatomy of the part.

The temporal lobe lies in the middle fossa of the base of the skull and is bounded above, anteriorly, by the Sylvian fissure. On its outer and convex surfaceo it presents three chief gyri, the superior middle and inferior temporal gyri. Ono the inferior surface there are three main gyri running approximately in an antero- $\frac{\bar{m}}{\overline{ }}$ posterior direction, namely the inferior temporal gyrus, the occipito-temporal gyrus, $\stackrel{\Phi}{\AA}$ and the Hippocampal gyrus, named from without inwards. The tip of the Hippos campal gyrus is close to the mesencephalon and pituitary, and is known as theuncus or uncinate gyrus. The medial aspect of the temporal lobe comes intocontact with the island of Reil. (Fig. I.)

The tracts in the temporal lobe consist of the auditory radiations, the terminato tion of the olfactory tract, and large association tracts which, in the left hemisphere 3 . in right-handed persons, are concerned with the function of speech. The auditoryor tracts arise from the internal geniculate body. The tracts arriving at the internal ${ }_{\circ}$ geniculate body are already so intermingled with those of the opposite side, that the auditory function is almost completely bilaterally represented. The auditory $\vec{\circ}$ radiations arise from the internal geniculate body, pass behind the optic radiationso in the internal capsule and diverge, in a fan-like manner, to end in the superiortemporal gyrus. Owing to the almost complete intermingling of the fibres in thes internal geniculate body, destruction of one temporal lobe very seldom gives rise to any perceptible impairment of hearing.

The olfactory tract, which arises in the olfactory bulb, ends in the Hippocamp? gyrus. Association tracts around the superior temporal gyrus are concerned wi ing speech and lesions of those tracts may give rise to sensory aphasia-word deafness. Deep in the temporal lobe, pass the fibres of the optic radiations which swervea outwards on their course from the external geniculate body to the occipital cortex. $\mathrm{D}$ These may be caught by a deep-seated temporal growth and give rise to partial? loss of the fields of vision, usually a quadrantic homonymous anopia.

We are now in a position to consider in detail the signs and symptoms produced by tumours of the temporal lobe.

Lesions in the region of the uncinate gyrus are commonly associated with characteristic Jacksonian attacks known as "uncinate fits". These consist of epileptiform attacks, with an aura consisting of an hallucination of taste or smell. The patient passes into a dreamy state, in which he smacks his lips, and performs rhythmical champing movements of the jaws. The sensation is invariably charac-o teristic and not varied in the individual. It is usually unpleasant, such as of burnings rubber or burning oil. The whole attack lasts only a few minutes and then passes off. Such attacks are diagnostic of a lesion of the uncinate gyrus.

As has been stated previously, lesions of the cortex of the temporal lobe doo not give rise to objective deafness, but on the left side in a right-handed person,, lesions damaging the association fibres of the auditory speech centre, will give rise? to verbal aphasia. If the lesion occurs in the posterior part of the temporal lobe, word-blindness may result. If it is situated deep to the angular gyrus, in addition to an homonymous hemianopia, a nominal aphasia may be found. In a patient suffering with nominal aphasia he can recognise objects presented to him, but is unable to name the object or to name it correctly. On his being told the right named 
of the object, a look of great satisfaction comes over the patient. He cannot say so, but he knows it to be correct. He may still, however, be unable to name the object himself, even after being told its name.

Deeply seated lesions involving the severance of the speech centre from the auditory tract, produces a condition known as " jargon aphasia ". Here the patient is unable to recognise the words he has spoken. Normally, speech is initiated on the motor side of the speech centre and the words spoken are "checked up " after being spoken by the auditory centre via the auditory tract. In these patients checking up does not occur and the words produced are disjointed, disconnected, and often meaningless.

Other changes produced by damage to the temporal lobe are a varying degree of mental impairment, and incontinence from loss of sphincter control. Pyramidal tract involvement may also be seen, evidencing itself in the loss of the abdominal reflexes on the opposite side, and later the production of an extensor plantar response.

The general signs produced are those of any cerebral tumour. There is headache, not usually localised to the side of the lesion. Vomiting occurs frequently and papillodema is quite often marked, usually more so on the affected side as would be expected. There may be itching at the nose and drowsiness, with mental impairment. Generalised fits may occur.

False localising signs are apt to arise. The pyramidal tracts may be pressed upon and give rise to a hemiplegia. The cerebellum may be involved, sometimes by direct extension on the side of the lesion, but more commonly due to pressure on the cerebellum, giving rise to ataxy and inco-ordination. The third and sixth nerves are commonly involved.

Diagnosis of these lesions is often very difficult as the false localising signs are apt to arise before the true localising signs.

The skull should always be X-rayed in these cases. Calcification occurs in certain varieties of cerebral tumour and also in cerebral abscesses which are apt to arise in that situation, and if of long standing, often calcify. Valuable information can also be obtained from the pineal, if calcified, as it is often shifted to the opposite side in these cases.

Lumbar puncture should also be performed and the pressure of the cerebrospinal fluid measured. Examination of the fluid may show the presence of cells or an increase of the protein content. The Wasserman reaction on both blood and cerebro-spinal fluid should be done in every case. A ventriculography is of considerable value as it will show distortion of the ventricular system with temporal lobe tumours of any size.

\section{Pathology.}

For the sake of completeness, abscess of the temporal lobe may be included in the term "tumours".

The middle ear gives rise to a large proportion of the lesions of the temporal lobe. Infection spreads through the temporal plate of the temporal bone, and gives rise to a cerebral abscess. If the extension is rapid then the signs of a cerebral tumour are produced. If the extension is slow, then a localised abscess is produced which has a thick wall and may calcify. These may remain latent for years and may be successfully removed by operation. 
Cholesteatomata may arise either in connection with the middle ear or quite apart from chronic otitis.

Meningiomata occur, usually on the surface, and are relatively easy to remove.

Neurofibromata arise either on the eighth nerve, where they may extend up into the temporal lobe and give rise to no physical signs at all, or on the fifth nerve, from which they may again extend up into the temporal lobe. These latter arise from the Gasserian ganglion and usually extend upwards into the Hippocampal gyrus.

Tumours arising from the nerve elements themselves, such as Astrocytomata and Spongioblastomata are the same as those occurring elsewhere in the central nervous system and do not require separate description here.

Secondary tumours also occur from malignant disease elsewhere in the body, and especial care should always be taken to exclude carcinoma of the bronchus, which is liable, more than other neoplasma to metastasise to the brain.

\section{Treatment.}

If the tumour is situated in the temporal pole, then lobectomy can be carried out with success, and it is curious to note that as this is a silent area, relatively large portions of this part of the brain can be removed without causing serious post-operative disability. The best form of anæsthesia is avertin, followed by intratracheal gas and oxygen. Local infiltration of the scalp is undertaken, using half per cent. novocain to which has been added some adrenalin solution.

A large horseshoe-shaped osteo-plastic flap is turned down and the dura matero exposed. If the intra-cranial pressure is much increased, an attempt is made too tap the lateral ventricle. Should this fail, owing to the fact that the ventricle iso compressed by the pressure exerted by the tumour, then the opposite ventricle is? tapped. The dura mater is opened as a flap and the brain exposed. If the tumour is situated in the temporal pole a lobectomy is carried out. The cerebral vessels are coagulated with the diathermy coagulating current. (Fig. 2.)

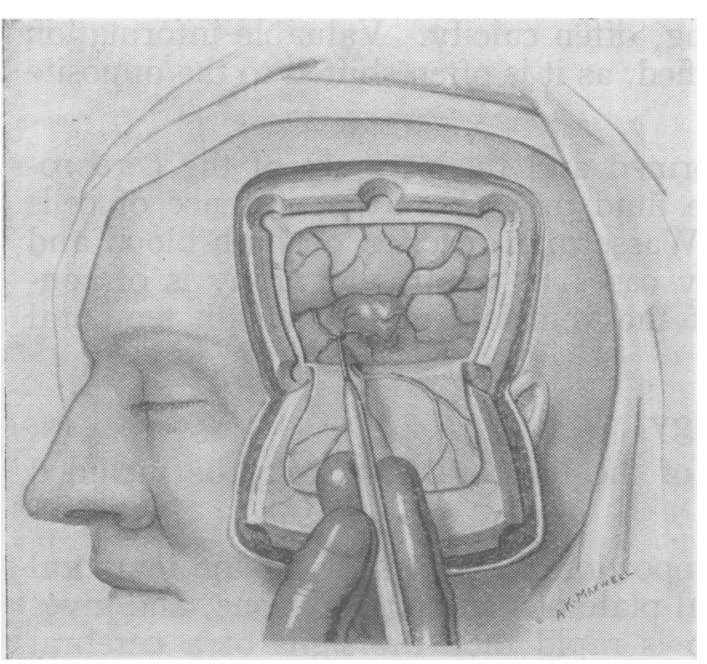

FIG. 2.-Drawing made at operation showing tumour in temporal pole. The cortical veins in the region of the tumour are coagulated with the diathermy cautery.

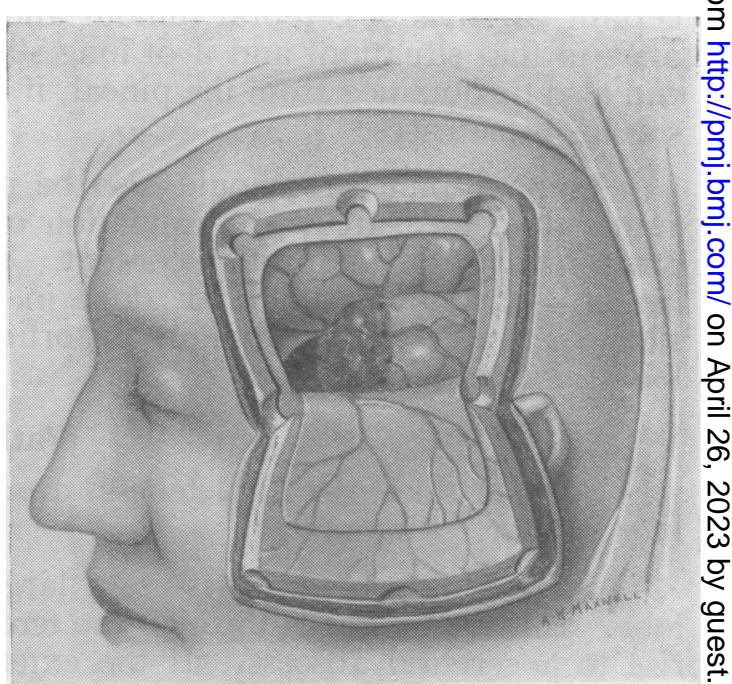

FIG. 3.-Appearance of the brain after lobectomy of the temporal pole for tumour. 
The area to be removed is decided upon and the cutting diathermy point is used to divide the brain substance, any big vessels encountered being coagulated "en route". The brain substance is separated by thin malleable retractors. The surgeon proceeds very slowly and carefully until the whole of the temporal pole in which the tumour is contained, is removed. The cut surface of the brain is examined to see that no sign of any tumour tissue remains and that all bleeding points have been effectively coagulated. (Fig. 3.) The dura mater is replaced and the osteo-plastic flap returned and sutured in two definite layers. No drainage is necessary as a rule. The patient is returned to bed and, if necessary, a small blood transfusion is given. As soon as consciousness is recovered he is nursed in the sitting position. Fluids are given liberally and solid food is taken about the third day. The patient is able to leave hospital within three weeks.

In those cases where the tumour is large and is situated in the substance of the temporal lobe, the best results are obtained by performing a palliative decompression followed by a course of deep X-ray therapy. I have obtained better results by this procedure than by partially removing the tumour and then giving $\mathrm{X}$-ray treatment.

\section{REFERENCES.}

CAVE, H. A. (1932), “Temporal Lobe Lesions; Disturbance of Visual Pathways ; Report of Cases," California \& West.

Med., 36, 13-18. Lobe," A. Research Nerv. \& Ment. Dis., Proc., 13, 251-258.

LESCHNER," M. RENDER, M. B. and STRAUSS, I. (1936), "Mental Symptoms in Cases of Tumor of Temporal Lobe," Arch. Neurol. \& Psychiat., 35, 572-596.

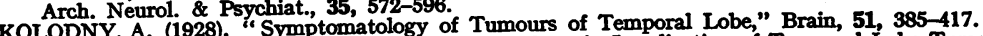

KOLODNY, A. (1928), "Symptomatology of Tumours of Temporal Lobe, Train, 51, 385-417. Tumors," Am. J. Ophth., 14. KRAVITZ, D. (1931), "Value of Quadrant Field Defects in Localization of Temporal Lobe Tumors," Am. OP. Ophth., 14. MEYERS, I. L. (1928), “Cerebellar Phenomena in Lesions of Temporal Lo Tumour of Posterior Temporal Region," Canad. M.A.J., 33, 32-36.

33, 32-36. 1 " Sub-total Removal of Tumour of Right Temporal Lobe," M. J. Australia, 2, 714-717.

PHLWTPS, G. (1936), " Verified Tumor of Temporal Lobe ; Critical Review of 52 Cases," Arch. Neurol. \& Psychiat., 30, 824-842. ROWE, S. N. (1933), "Verified Tumor of Temporal Lobe ; Critical Re Temporal Lobe," Arch. Neurol. \& Psychiat., 29, 843-854 SCHIESINGER, B. (1933), "Syndrome of Fibrillary Astrocytomas of Temporal Lobe, Arv. \& Ment. Dis., 79, 1-13. 